\title{
GEOGRAFÍA Y CRECIMIENTO URBANO. PAISAJES Y PROBLEMAS AMBIENTALES
}

\author{
PROF. JUAN A. ALBERTO
}

Departamento e Instituto de Geografía. Facultad de Humanidades. UNNE. Resistencia. Chaco. Argentina

E-mail: albertoja@arnet.com.ar l ja_alberto@yahoo.com

\section{RESUMEN}

El presente escrito surge del soporte teórico y apreciaciones prácticas sobre el fenómeno de expansión urbana y sus paisajes y problemas derivados, fundamentos que en el trabajo de investigación que realizo en el Departamento e Instituto de Geografía, de la Facultad de Humanidades, UNNE, están acotados al Área Metropolitana del Gran Resistencia, en la Provincia del Chaco.

Es incuestionable, hoy, que el vertiginoso crecimiento urbano y la multiplicación de ciudades, en los últimos cincuenta años, es un fenómeno social y demográfico con un fuerte impacto sobre el ambiente. El hacinamiento de personas en áreas de riesgos ambientales (inundaciones, vendavales, sismos, etc.) acrecienta su vulnerabilidad y hacen que los factores ambientales sean una de las principales causas de muerte, enfermedad y pérdida de la productividad, preservando la pobreza. Probado está que los procesos de urbanización son las transformaciones más radicales que el hombre inflige sobre el entorno, tal vez uno de los impactos más agresivos acompañado de una diversidad de problemas ambientales a veces impredecibles, ya que por un lado al extenderse sobre los ecosistemas naturales originales los degradan generando un nuevo ambiente: la ciudad, un ecosistema artificial, calificado como parásito por muchos entendidos en el tema, pues toma energía y recursos varios de otros ecosistemas, vecinos en algunos procesos, lejanos en otros. Por otro lado generan un cúmulo de desecho que contaminan y menoscaban la calidad del suelo, aire y agua del sitio que ocupan y de espacios circundantes (periurbanos y rurales).

Está claro que los resultados de estos procesos e interacciones, ya sean de orden natural o antrópico configuran en el espacio un mosaico de fisonomías que denominamos paisajes, caracterizadas en estos espacios y áreas contiguas por un fuerte dinamismo que imprime rápidos cambios en sus componentes, tanto físicos, biológicos como humanos.

\section{PALABRAS CLAVES:}

Geografía, crecimiento urbano, vulnerabilidad, problemas ambientales, paisajes.

\section{GEOGRAPHY AND URBAN GROWTH. LANDSCAPES AND ENVIRONMENTAL PROBLEMS}

\section{SUMMARY ${ }^{1}$}

\footnotetext{
${ }^{1}$ Traducción al inglés Prof. José Manuel Monserrat Castillo - Dpto. de Lenguas Extranjeras. Fac. Humanidades.UNNE
} 
This work is the result of the theoretical framework and practical appraisal of the phenomenon of urban sprawl, its landscapes and related problems as part of the research I carry out in the Department and Institute of Geography, Faculty of Humanities, UNNE, and which is limited to the Metropolitan Area of Resistencia in the province of Chaco.

The rapid growth of towns and cities during the past fifty years is, without doubt, a present social and demographic phenomenon with a strong impact on the environment. The overcrowding of people in areas of environmental hazards (floods, windstorms, earthquakes, etc...) increases their vulnerability and turns the environmental factors into a major cause of death, disease and loss of productivity, while retaining poverty.

It has been proved that the processes of urbanization are the most radical transformations that man inflicts on the environment, perhaps one of the most aggressive impacts which imply a variety of environmental problems, sometimes unpredictable, since the expansion over the natural ecosystems, on the one hand, degrades them producing a new environment: the city, an artificial ecosystem, featured by many as a parasite. This is so because the city takes energy and several resources of other ecosystems, which share the same processes, while in some others they are just the opposite. On the other hand it generates a lot of waste that pollutes and undermines the quality of soil, air and water, not only of the site it occupies but also surrounding areas (suburban and rural).

It is clear that the results of these processes and interactions, whether natural or artificial, make up in space a mosaic of physiognomies that we call landscapes, characterized in these spaces and adjacent areas by a strong dynamism that imprints rapid changes in its physical, biological and human components.

\section{KEY WORDS:}

Geography, urban growth, vulnerability, environmental problems, landscapes.

\section{A MANERA DE INTRODUCCIÓN}

La Geografía y la Ecología del Paisaje han adquirido en las últimas décadas mucho prestigio y han sido adoptadas, en los países desarrollados, por planificadores y gestores de la administración pública y privada, involucrados en la toma de decisiones acerca del manejo del espacio con fines agropecuarios, forestales, industriales, de conservación de la vida silvestre, pero especialmente en todo lo que atañe a los ámbitos urbanos.

Al respecto, el acelerado crecimiento urbano y la proliferación de ciudades es, tal vez, una de las preocupaciones de mayor interés para estas nuevas áreas del saber, pues tienen que ver con los fenómenos sociales y demográficos más sobresalientes de la segunda mitad del siglo XX y lo que va del actual; ya que las urbes, como ecosistemas artificiales o antrópicos, son espacios reducidos que se caracterizan, desde lo social, por concentrar una alta densidad de población, sustentada por la inmigración del campo a la ciudad, y de poblados y urbes pequeñas a centros y metrópolis importantes, a lo que se le debe sumar el incremento por crecimiento natural propio; mientras desde lo ambiental se destacan por la fuerte demanda y entrada de energía y recursos naturales para su funcionamiento y existencia y, como consecuencia de ello, la posterior salida de productos elaborados y una gran cantidad de desechos.

Está claro que la urbanización como proceso, tanto en número de urbes como en cantidad de habitantes, es un fenómeno social y demográfico con un fuerte impacto sobre el ambiente. El hacinamiento de personas en áreas de riesgos ambientales (inundaciones, vendavales, etc.) aumenta su vulnerabilidad y hacen que los factores ambientales sean una de las principales causas de muerte, enfermedad y pérdida de la productividad, perpetuando la pobreza. Ante esto, a la hora de analizar las relaciones ambiente y sociedad como también la pérdida del equilibrio dinámico de la naturaleza, es preciso tener muy en cuenta el crecimiento y expansión de las ciudades, ya que el uso del suelo con fines urbanos es irreversible. 
Probado está que los procesos de urbanización son las transformaciones más radicales que el hombre inflige sobre el entorno, tal vez uno de los impactos más agresivos, ya que por un lado degradan los ecosistemas naturales originales al expandirse sobre ellos generando un nuevo ambiente: la ciudad, un ecosistema artificial, considerado parásito por muchos entendidos en el tema, pues toma energía y recursos varios de otros ecosistemas, vecinos en algunos procesos, lejanos en otros. Por otro lado generan un cúmulo de residuos que contaminan y menoscaban la calidad del suelo, aire y agua del sitio que ocupan y de espacios circundantes (periurbanos y rurales).

No hay duda que los resultados de estos procesos e interacciones, ya sean de orden natural o antrópico, plasman en el espacio un mosaico de fisonomías que denominamos paisajes, caracterizadas en estos ámbitos, y sus áreas adyacentes, por un fuerte dinamismo que imprime rápidos cambios en sus componentes, tanto físicos, biológicos como humanos [Fig. № 1].

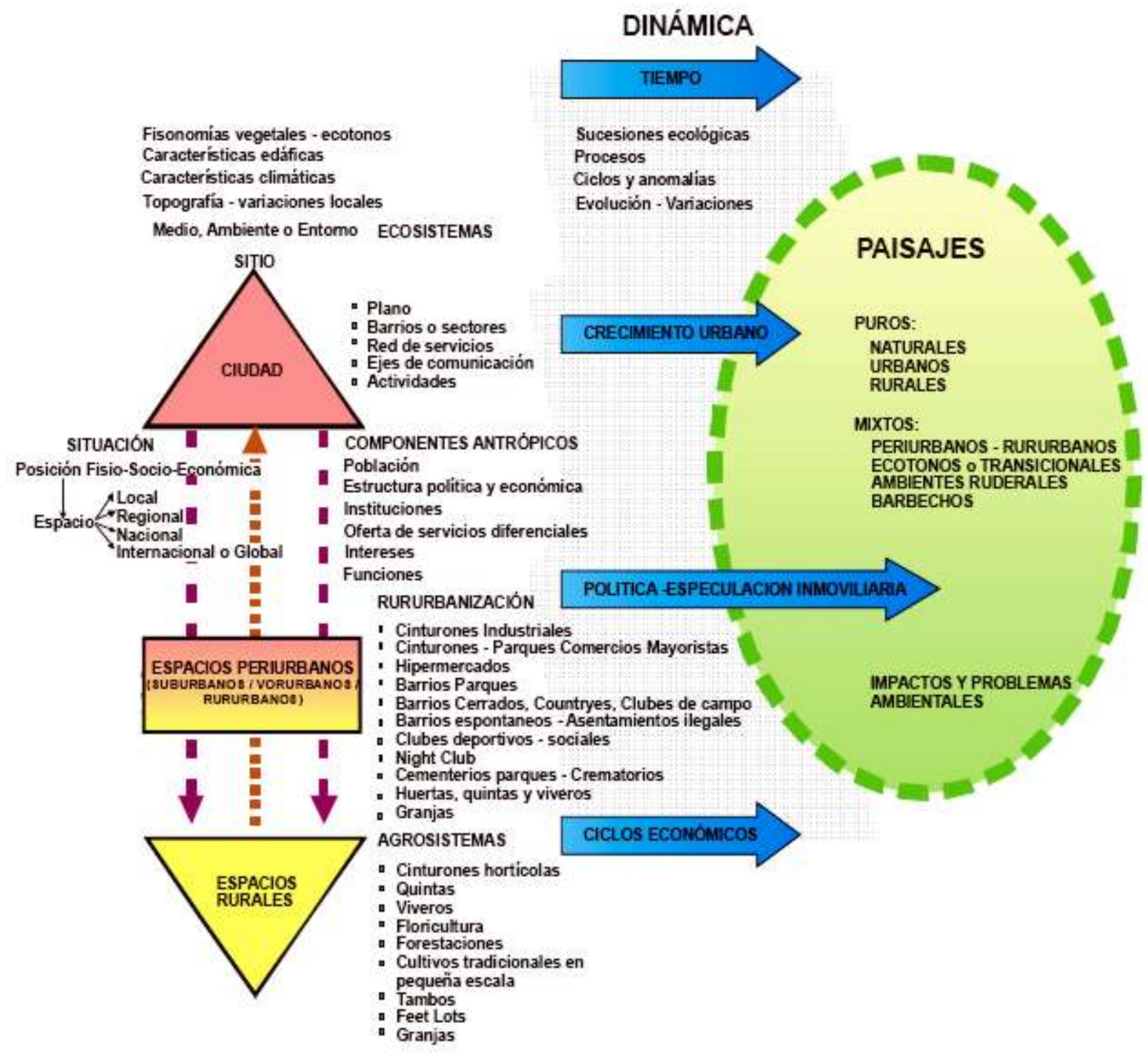

Prof. Juan Antonio ALBERTO. IGUNNE. Chaco. Argentina. XI Encuentro de Gećgráas de América Latina. 'Caminando en una América Latina en transformación' 3 al 7 de abril de 2009. Montevideo, Uruguay

\section{Fig. 1: Expansión Urbana, Paisajes y Dinámica}




\section{EL PAISAJE Y EL DINAMISMO DE SU CONFIGURACIÓN}

En la naturaleza cada espacio es ocupado a través del tiempo por diversas biocenosis o comunidades vegetales y animales, las que son influidas por diversos factores ambientales, y especialmente por las múltiples interacciones que se dan entre sus componentes abióticos y bióticos. Esta evolución y variación de las diferentes comunidades de vida y los aspectos del entorno conforman un proceso denominado sucesión ecológica, y como resultante de esto se dan distintos paisajes o fisonomías geográficas para cada etapa o sere.

Paralelos a las evoluciones inherentes a la naturaleza, se dan los procesos de ocupación y organización del espacio por los distintos grupos humanos, generando sus propios paisajes con sus impactos y problemas ambientales asociados. [Fig. № 2]

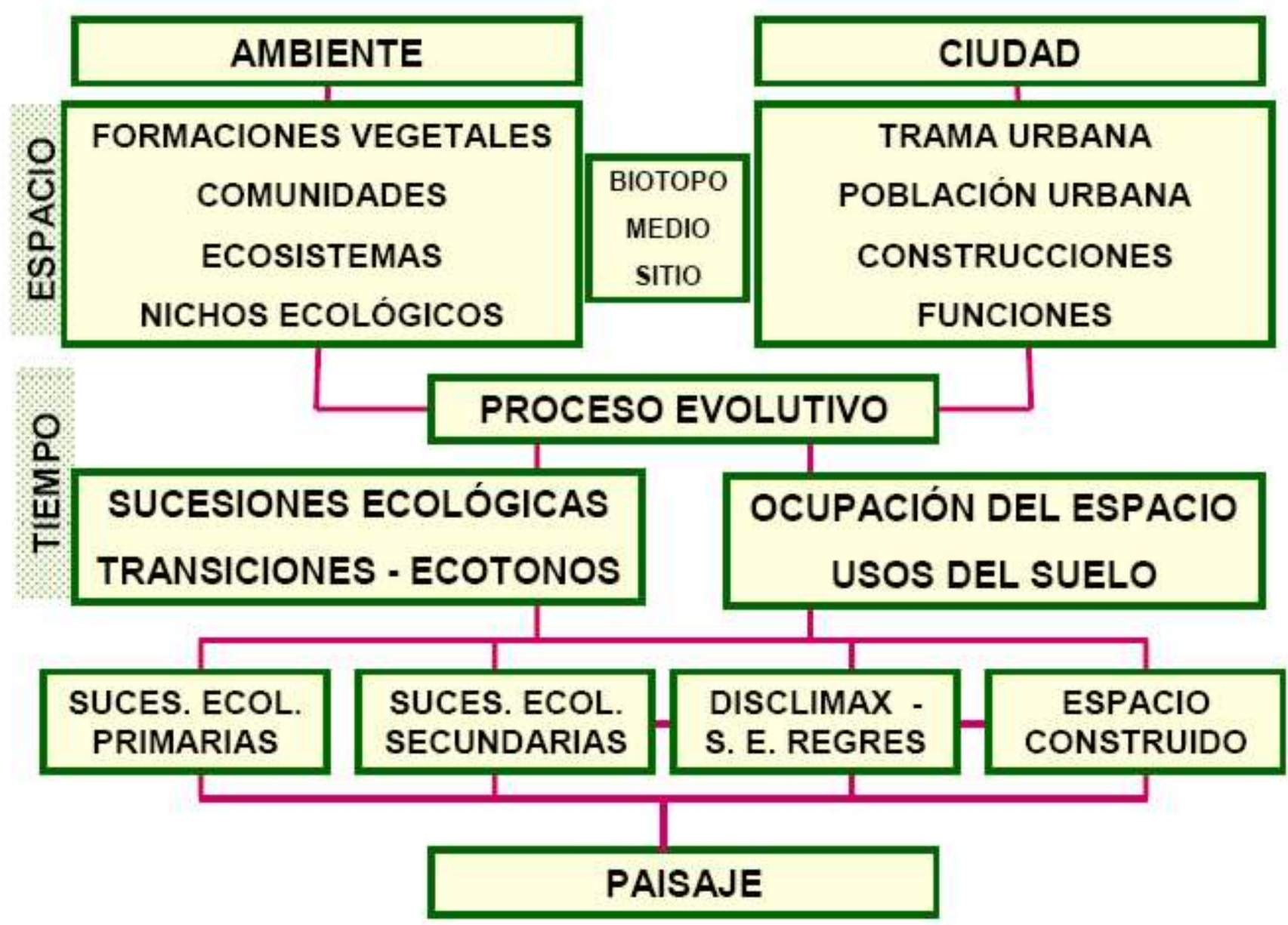

Fig. № 2. Componentes y Dinámica Espacio Temporal del Paisaje

La Convención Europea del Paisaje (2000) en su Capitulo I, expresa que: "El paisaje designa cualquier parte del territorio, tal como es percibida por las poblaciones, cuyo carácter resulta de la acción de factores naturales $y / 0$ humanos $y$ de sus interrelaciones;...". Podríamos agregar que el espacio geográfico se nos presenta como un conglomerado de estampas resultantes de procesos e interacciones, ya sean de orden natural o antrópico, y que según el predominio de componentes naturales o artificiales serán las percepciones que tengamos de ellos, como paisajes naturales por ejemplo las regiones polares o de altas montañas, lugares recónditos y raros de selvas, desiertos e islas; en el extremo opuesto veremos comarcas 
con una mayor proporción de elementos construidos por los grupos humanos, es decir paisajes culturales o humanizados, donde los elementos naturales serán progresivamente reemplazados por componentes artificiales, por ejemplo campos o áreas de cultivos y actividades ganaderas, yacimientos de extracción de minerales, pueblos, ciudades, metrópolis y parques industriales, centros de deportes invernales y estivales, etc.

En el caso de la ciudades y sus áreas adyacentes, estas fisonomías paisajísticas son frutos de un complejo ínter juego de variables, que van desde la propia historia del núcleo urbano central y sus vecinos, de la complicada geografía del sitio donde fue asentado y creció, de las políticas de turno, de los ciclos económicos y de su situación en el mapa regional, nacional e internacional. Representaciones que varían en el tiempo y en el espacio (urbano, suburbano, periurbano, vorurbano, rururbano, rural, natural) tanto en hechos concretos, como en la percepción que tienen de estos paisajes cada uno de los actores sociales implicados, de acuerdo con sus propios intereses y paradigmas. [Fig. № 3]

\section{CRECIMIENTO URBANO Y DINAMIZACIÓN DEL PAISAJE Paisajes Puros, Paisajes Mixtos y Transiciones}

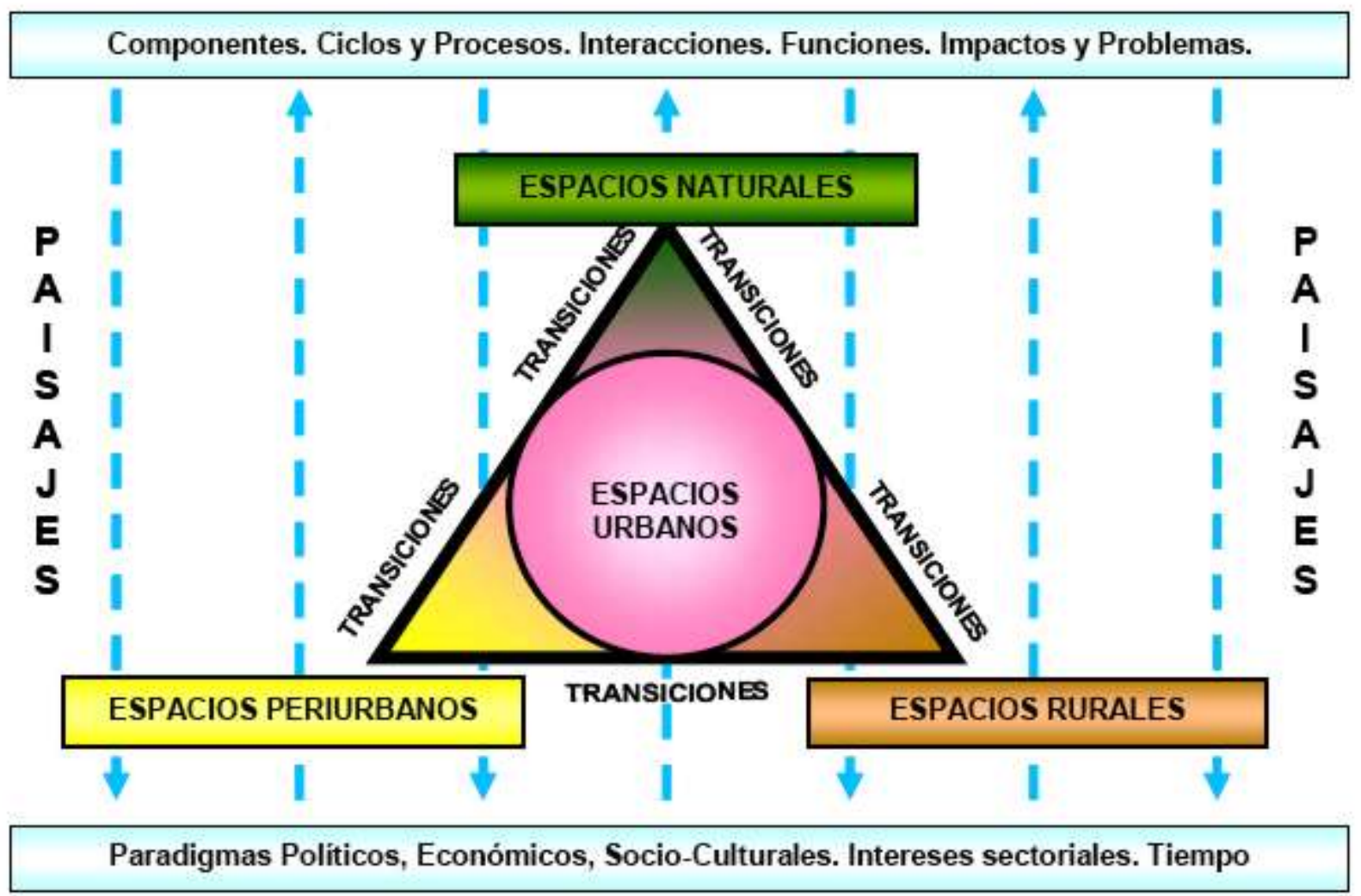

Prof. Juan Antonio ALBERTO. CRECIMIENTO URBANO Y DINAMIZACIÓN DEL PAISAJE. En "X Jornadas Cuyanas de Geografia", Universidad Nacional de Cuyo. Mendoza. 28 at 31 de mayo de 2008

Fig. $N^{\circ}$ 3. Crecimiento Urbano y Paisajes Derivados

Es decir que cada paisaje tiene un dinamismo propio que imprime constantes variaciones en sus componentes, en su configuración y en su apariencia, energía ésta impulsada por fuerzas endógenas (propias del paisaje, como componentes ambientales del sitio, características de la situación -a diversas 
escalas- del paraje en cuestión sumada a la acción de los grupos humanos que lo ocupan y organizan) y exógenas (exteriores, provenientes de espacios cercanos o lejanos, con variada magnitud y constancia, tales como políticas de tierras, estrategias económicas a nivel regional, nacional e internacional, como la globalización misma).

Indudablemente que el proceso de urbanización, es unos de los vectores más dinámicos al configurarse aquellos paisajes netamente antropógenos, ya que las transformaciones producidas por este fenómeno generan continuos y acelerados cambios en la fisonomía de un espacio dado, visibles en un par de lustros o décadas, muy especialmente en los últimos 40 años. Obviamente que, estos impulsos, estarán acordes al rango de cada centro urbano y su trascendencia en la vida regional, nacional e internacional. Las ciudades o conurbanos con rango de metrópolis regionales nacionales o continentales, son las que muestran mayores variaciones y una mayor dinamización de su paisaje y el de sus comarcas vecinas, sean estas ambientes naturales, espacios periurbanos (suburbanos, vorurbanos, rururbanos) y/o áreas rurales, con toda una gama de fisonomías mixtas y ecotonos o paisajes transicionales. Claro esta que cada uno de estos paisajes comparten, con mayor o menor rigor, las huellas o impronta de la ocupación y organización del espacio por los grupos humanos a través de distintos usos del suelo, los que se traducen primero en un impacto ambiental, y en unos cuantos años en problemas ambientales, de ser estas actividades agresivas y constantes al no respetar tiempos, ciclos y procesos naturales del entorno.

La valoración del paisaje, aunque parece sustentarse en ciertos aspectos instintivos, es esencialmente cultural y aprendida; puede ser mejorada o afianzada y, por consiguiente, también enseñada. Distintas civilizaciones y culturas han tomado en consideración el paisaje en diferentes momentos históricos y lo han cargado de sentidos o matices particulares; en unos casos se pone mayor énfasis sobre aspectos ambientales, en otros sobre los sensoriales, saludables o estéticos. Pero actualmente la existencia de una palabra que lo designa expresamente, su representación icónica con el propósito de transmitir las peculiaridades de un lugar o los valores generales en él percibidos están presentes en todas las sociedades. En el aprecio social del paisaje reside la causa principal para convertirlo en un derecho individual, premisa sustentada en una de las ideas más vivamente propagadas durante las tres últimas décadas del siglo XX, el anhelo de vivir en un ambiente digno y sano.

\section{URBANIZACIÓN, PAISAJES Y PROBLEMAS AMBIENTALES}

Frente al proceso de urbanización y su dinámica se establece un nuevo sistema de relaciones sustentado por nuevos parámetros funcionales (ambientales, demográficos, de flujos, de usos del suelo, etc.) que surgen y se nutren de vínculos, avances, roces, conflictos e interdependencias entre la ciudad y el campo, entre esta y su periferia, y entre esta última y el ámbito rural y toda una gama de transiciones entre lo urbano, suburbano, rural y natural.

En la actualidad, en cualquier ciudad intermedia, grande o conurbano, la identificación de límites y fronteras entre el ámbito natural no urbanizado y el espacio urbanizado es difícil, puesto que el atributo urbano se ha expandido de modo discontinuo, de acuerdo con el fenómeno denominado paraurbanización y que puede tener carácter periurbano, rururbanoo vorurbano. En las periferias urbanas, precisamente, aparecen multitud de nuevos usos que no se pueden calificar ni de propiamente urbanos ni de exactamente rurales tales como invernaderos y quintas semiurbanizadas, equipamientos diversos, campus universitarios, almacenes mayoristas, clubes, cementerios parques, etc. (periurbanización); las áreas naturales protegidas por sus valores paisajísticos y ecológicos, como una alta biodiversidad, presencia de rarezas y endemismos, etc., restan como fragmentos aislados en la matriz periurbana y frecuentemente están sometidas a intensas presiones.

Además, del mismo modo pueden encontrarse complejos turísticos, industrias aisladas, centros de investigación, complejos militares, etc., en ámbitos rurales, así como grandes infraestructuras (rururbanización). Por último, hay todo tipo de restos paisajísticos en los resquicios de las grandes infraestructuras viales, energéticas, etc., sentenciados a ser finalmente extinguidos por el espacio periurbano (vorurbanización). 
Al respecto, valen aquí las apreciaciones de Neil Smith (1996) quien sostiene que la ciudad contemporánea vive grandes transformaciones tanto en sus límites exteriores como en su espacio interno, unas mutaciones que subrayan época por cuanto cambian las relaciones socioespaciales y sus formas, por ende sus paisajes derivados. Para Joan Vicente Rufí (2000) es este urbanista quien recupera las conceptualizaciones del geógrafo franco-norteamericano Jean Gottmann (1961) que, en los años sesenta del siglo XX, expresaba "la nueva frontera es la urbana y la suburbana", investigador que junto con otros autores como Jane Jacobs y Lewis Mumford, observó el proceso de suburbanización de la sociedad de los Estados Unidos como un nuevo modo de colonización y de "conquista" de territorios "vírgenes". Una continuación del mito de la frontera - del go west - tan presente en la historia de ese país. N. Smith, en los umbrales del siglo XXI, revaloriza y recupera la expresión de "la nueva frontera urbana y suburbana" aplicándola a la ciudad contemporánea, signada por los cambios tecnológicos, por la globalización de la economía, por las migraciones y por la puesta en cuestión de los modelos urbanos y urbanísticos determinantes de la segunda mitad del siglo pasado.

Indudablemente que estos cambios paisajísticos connotan modificaciones tantos estructurales o de componentes e interacciones tanto en los ecosistemas naturales del sitio como en los ecosistemas culturales surgidos de la ocupación y organización del espacio por el grupo humano que da origen al asentamiento urbano. Se aprecia entonces, en la actualidad, que el avance de las edificaciones, las calles, las redes de servicios (agua, luz, cloacas) impactan sobre el medio y alteran considerablemente el paisaje natural original, en nuestro caso específico [Área Metropolitana del Gran Resistencia (AMGR)] algunos de los ejemplos más notables son la degradación y la destrucción de los espacios naturales, la colmatación de cuencas (lagunas, ríos y bajos) y la proliferación de basurales a cielo abierto, de incorrecta ubicación, que generan problemas socioambientales graves como focos ígneos de compuestos tóxicos, generación y propagación de vectores de enfermedades infecciosas, contaminación de suelos y napas freáticas, y además el surgimientos de barrios de excluidos sociales, que viven de "cirujeo" en sus proximidades.

Esta problemática responde, como en otras partes del mundo, según los responsables del Proyecto LIFE de la Comunidad Económica Europea a que "los cambios que se han producido en los hábitos de consumos, unidos a la desidia y la falta de sensibilidad ambiental de los ciudadanos, han originado por una parte un aumento en la producción de residuos y por otro la aparición de vertederos incontrolados distribuidos alrededor de los núcleos de población. Esta situación unida a que la mayor parte de los vertederos municipales o mancomunados no cumplen las mínimas medidas de control a originado una disminución de la calidad ambiental"....

A esto podemos agregar citando Arnaud, A. (2000) que la cantidad promedio de residuos son muy variables, pero termino medio para un habitante de una ciudad de país desarrollado es de $1 \mathrm{~kg}$ de desecho por días, y según Bosch, D. (1998) en el Área Metropolitana del Gran Resistencia [conurbano motivo del estudio que forma parte este escrito] en 1996 representaba $0,46 \mathrm{~kg}$ al día por persona, que equivaldrían para el municipio de Resistencia unas 120 toneladas diarias, incluyendo residuos domésticos, comerciales y del barrido de calles.

Bien vale recordar, como una aseveración más de lo analizado hasta aquí, la opinión del arquitecto panameño Jorge Ricardo Riba (2007) quien dice "Si en algo se asemejan las grandes ciudades de América Latina es en su arquitectura globalizada y altos edificios los cuales no pueden esconder las evidencias de la pobreza, así se empuja hacia la periferia a grandes núcleos de población carentes de los esenciales servicios básicos en un vano intento de diluirla en el paisaje.

El urbanismo y su correspondiente infraestructura, las áreas públicas, transporte, los servicios comunales, los parques con las normas apropiadas se ignoran. Se asesina ecológicamente el paisaje natural ya que el lucro es lo que impera."

Claro está entonces, especialmente en las últimas décadas, que el crecimiento sostenido, rápido y generalmente desordenado de los centros urbanos sin medidas mínimas de ordenamiento urbano que respetaran las peculiaridades del sistema natural, han generando problemas ambientales, sociales y paisajísticos notorios, lo cual adquiere un dramatismo singular, pues el uso del suelo con fines urbanos es irreversible. 


\title{
Conclusiones:
}

Frente a la problemática aquí analizada, podemos adelantar algunas consideraciones y premisas a ser tenidas en cuenta al estudiar y buscar alternativas de solución a los diferentes conflictos surgidos de la relación e interacción de los grupos humanos y el ambiente, en el cual configuran el espacio geográfico con sus distintas fisonomías o paisajes. Juzgando que más allá de los paradigmas de moda e ideologías imperantes, ambos componentes básicos y críticos no pueden ser ignorados ni excluidos en beneficio del otro, menos en beneficios de sectores hegemónicos desde el punto de vista económico y político, hecho este último que va en desmedro del ambiente y de la sociedad toda en última instancia, no olvidando que alteraciones inflingidas al entorno vuelven a los grupos humanos como problemas que van de simples trastornos a grandes y lamentables catástrofes, y que siempre es más barato prevenir que revertir dichas acciones negativas. En definitiva deberíamos propender a:

1- La búsqueda de equilibrio entre lo Social y lo Ambiental, hacia un desarrollo urbano sostenible

\author{
OCUPACIÓN Y ORGANIZACIÓN DEL ESPACIO POR LOS GRUPOS SOCIALES<smiles>C1CC2CCCC2C1</smiles> \\ $\checkmark$ utópico pensar detener el crecimiento de la población y la expansión urbana, \\ $\checkmark$ imposible dejar de producir alimentos y distintos recursos esenciales para la vida, \\ $\checkmark$ inadmisible no dar vivienda y vestimenta adecuada al ecumene \\ No debe ser el dilema "Sociedad o Ambiente" \\ Transformemos al dilema en problema y \\ al problema busquémosle soluciones. \\ búsqueda del equilibrio perdido \\ Usar todo el cuerpo de conocimientos científicos y tecnológicos \\ Hacer un mejor uso del entorno y los recurso que el nos brinda \\ Respetar sus ritmos y ciclos naturales para no caer en \\ desequilibrios y acrecentar la vulnerabilidad socio ambiental \\ Registrar y valorar la riqueza paisajística, tanto natural \\ como cultural para su preservación reglamentada \\ Gestionar una adecuada política y educación ambiental en pos \\ de una cultura ambiental
}


Prescripción y aplicación de normas ambientales

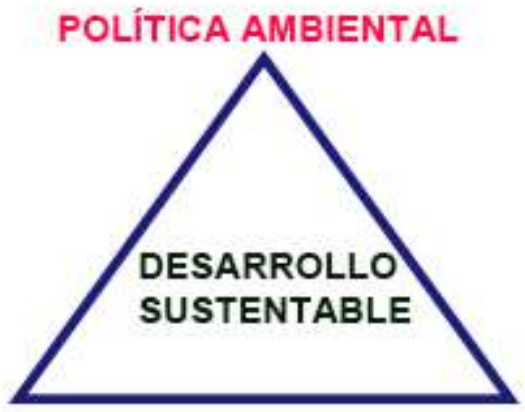

\section{EDUCACIÓN AMBIENTAL}

Formación e información para una mejor interacción en y con del ambiente

\section{CULTURA AMBIENTAL}

1) un estilo de vida de convivencia armónica "en y con" el entorno; 2) la defensa del patrimonio ambiental, histórico y socio cultural de su ámbito de residencia (riqueza paisajistica); 3) la búsqueda permanente de alternativas que permitan un mejor conocimiento y uso sostenible del ambiente

\section{PLANES ESTRATEGICOS DE DESARROLLO URBANO SOSTENIBLE}

\section{2- La Interpretación de la pluricausalidad del dinamismo urbano y sus paisajes y problemas consecuentes}

También valen aquí algunas reflexiones y recomendaciones sobre tratamiento y evaluación del crecimiento y expansión urbana, sus paisajes, impactos y problemas ambientales resultantes. Al respecto considero que es primordial e ineludible:

1- Justipreciar que las transformaciones del paisaje, debidas a la expansión del fenómeno urbano en el territorio y al desarrollo de la capacidad tecnológica de transformación de la naturaleza, no son nuevas, pero en las últimas décadas han alcanzado un ritmo antes desconocido, según lo expresa Busquets, Jaume (2004); quien acota que "...en la mayoría de casos, el resultado de estas transformaciones se manifiesta en la generación de paisajes estandarizados e impersonales, dando lugar al fenómeno conocido como "banalización del paisaje".

2- Conocer y darle valor a los fenómenos naturales como variables condicionantes del crecimiento urbano y de la configuración de paisajes, como también de la dinámica propia de los mismos.

3- Revalorizar y remarcar procesos, etapas y ciclos evolutivos, tanto en lo natural como en lo antrópico, del sitio y la situación del centro urbano estudiado, para explicar el dinamismo de éste y su estado actual y prever situaciones futuras.

4- Dimensionar la incidencia de ciclos económicos y políticas regionales, nacionales y globales sobre el crecimiento de un sitio en cuestión, como también en su dinamismo y evolución, que concluyen plasmados en sus paisajes consecuentes.

5- Valorar la presión de la especulación inmoviliaria, medidas políticas locales y reivindicaciones sociales en la evolución de la mancha urbana, ejes de crecimiento urbano, desaparición de espacios naturales, transformación y plusvalía de áreas rurales periféricas y la dinámica general del paisaje. 
6- Analizar al urbanismo como un proceso complejo según Tella, Guillermo (2007), remarcando que la administración pública, en beneficio de todos los ciudadanos, debe negociar políticas y cambios con los diferentes agentes urbanos, cada uno de los cuales defiende sus intereses, y actuar como árbitro objetivo entre sus conflictos y diferencias.

7- Asumir la aparición de problemas urbanos, tan inéditos como extremos, tales como la miseria urbana, el incremento sustancial de la pobreza extrema y su segregación física que están alcanzado niveles hasta ahora desconocidos.

8- Tender a una gestión de la ciudad donde el planeamiento propenderá a incorporar de manera gradual a la ciudadanía en todo su proceso de desarrollo, de tal forma que al incluir lineamientos corporativos en un extremo y sectores de mayor representatividad en el otro, se consoliden las políticas territoriales, por un lado y las expresiones emergentes del interés público, por el otro.

9- Por último, como lo dice Guillermo Tella, pensar que no hay estética sin ética. El urbanismo, en general, se ha elaborado e impuesto desde arriba hacia abajo, por ello se hace necesario un repensamiento del mismo basado en el diálogo con las partes implicadas evitando la prepotencia de los técnicos y su pretensión de que son poseedores exclusivos del saber.

\section{3- Redimensionar la Geografía, su trascendencia y aportes}

Hoy, sin duda frente a lo planteado brevemente hasta aquí, considero a manera de conclusión que es primordial conocer qué ocurre en el espacio para poder diagnosticar, planificar acciones futuras y programar estrategias de acción. Es por ello que desde la perspectiva del avance del conocimiento científico y tecnológico, especialmente en nuestro caso desde la visión de la Geografía Ambiental, la Geografía y Ecología del Paisaje se podrán proyectar y emprender trabajos que ayuden a proporcionar una base general para el tratamiento de la información sobre la historia ecológica de cualquier ciudad y sus zonas aledañas que se aborde, asimismo la tipificación y caracterización de sus paisajes, como también conseguir perfilar tendencias paisajísticas según la evolución de los procesos naturales y humanos estudiados, es decir su dinámica. No está demás remarcar la trascendencia del saber geográfico y la Geografía como ciencia en este tipo de estudios, ya que en ellos confluyen los aportes de las distintas especialidades de nuestra ciencia (físicos, biológicos o ambientales y humanos), a la vez que nos remiten a nuestros cuatros principios rectores, localización, causalidad, conexión y actividad o dinámica, que dan sustancia y seriedad a cualquier juicio que emitamos sobre esta temática.

En la actualidad paralelos a la trascendencia de los temas ambientales, los estudios de los paisajes cobran mayor vigencia y presencia dentro del amplio espectro de saberes interesados en un mejor uso del espacio. Recordemos que la percepción y valoración del paisaje, aunque parece sustentarse en ciertos aspectos instintivos, es esencialmente cultural y aprendida; puede ser mejorada o afianzada y, por consiguiente, también enseñada; es así que, desde esta óptica, nuevamente la Geografía como ciencia y disciplina, cobra relevancia ya que a través de la producción científica y de sus aportes curriculares al proceso de enseñanza y aprendizaje indudablemente enriquece a la educación general y a la educación ambiental, en particular, al aportar contenidos y herramientas para abordar y orientar los estudios ambientales y del paisaje, y por ende mejorar su percepción incentivando un uso racional de los recursos del entorno y la preservación del patrimonio paisajístico tanto natural como cultural.

\section{BIBLIOGRAFÍA}

- Aguirre Madariaga, Eduardo (1999-2003) Lagunas vs. Asentamientos. En ECO Portal - Net: www.ecoportal.net/content/view/full/21321

- Aguirre Madariaga, Eduardo (2004). ¿Por qué nos inundamos en Resistencia? El costoso error de seguir rellenando lagunas. Revista Chaqueña. Diario Norte. 29/04/2004. Pag.18

- Alberto, Jorge A. (2002). Análisis evolutivo de los usos el suelo del espacio periurbano del Gran Resistencia.XXII Encuentro de Geohistoria Regional. Instituto de Geohistoria Regional (IGHI) en la Ciudad de Resistencia el 4 y 5 de Octubre del 2002. 
- Alberto, Juan Antonio (2009). "Geografía, Crecimiento Urbano, Ambientes, Paisajes y Problemas. El Área Metropolitana Gran Resistencia". En: $12^{\circ}$ Encuentro de Geógrafos de América Latina. Montevideo, Uruguay. Easy Planners. ISBN 978-9974-8002-8

- Alberto, Juan Antonio (2008). Crecimiento Urbano y Dinamización del Paisaje. En. X Jornadas Cuyanas de Geografía. Universidad Nacional de Cuyo. Mendoza. ISBN 978-950-774-145-6.

- Alberto, Juan Antonio (2008). Crecimiento Urbano, Paisajes y Problemas. En: $2^{\circ}$ Seminario sobre Políticas Urbanas, Gestión Territorial y Ambiental para el Desarrollo Local. IPUR Facultad de Arquitectura y Urbanismo - UNNE. Resistencia, Chaco. ISSN 1851-3506.

- Alberto, Juan Antonio (2007). "Vulnerabilidad Ecológica y Natural. El Caso del Área Metropolitana Gran Resistencia". En: "Aportes conceptuales y empíricos de la vulnerabilidad global". Foschiatti, Ana María (2007). Autora-Compiladora. Resistencia, EUDENE, UNNE. Capítulo IV. ISBN 978-950-656-108.

- Alberto, Juan Antonio (2006). Las Fisonomías Vegetales del Chaco Oriental. En Revista Geográfica Digital, ISSN № 1668-5180. № 5, Año 3, Enero - Junio 2006.

- Alberto, Juan Antonio (2006). "En busca del equilibrio perdido: Ambiente o Sociedad... Ambiente y Sociedad. El Caso del Área Metropolitana del Gran Resistencia". En Comunicaciones Científicas y Tecnológicas 2006. Humanidades. Resumen: H-013. 4p.p. En: http://www.unne.edu.ar/Web/cyt/cyt2006/index.htm

- Alumni, José (1950). La Ciudad de Resistencia. Apuntes Históricos. Resistencia.

- Arnau, Arturo (2000). El medio ambiente. Problemas y soluciones. Miraguano Ediciones. Madrid (España).

- Bolós i Capdevila, María de y otros (1992). Manual de Ciencia del Paisaje. Teoría, métodos y aplicaciones. Masson S.A. Barcelona

- Bruniard, E. (Director). Geografica ${ }_{2}$ Revista del Instituto de Geografía, Facultad de Humanidades, UNNE, No1 (1972), No2 (1973), No3 (1974), № 4 (1975-78), Facultad de Humanidades, Resistencia.

- Bruniard, E. (1975-78). El Gran Chaco Argentino. (Ensayo de interpretación geográfica). En: Geográfica, Revista del Instituto de Geografía Nº4, Resistencia, UNNE.

- Bruniard, E. (1987).Geografía del Nordeste argentino. Buenos Aires, SENOC.

- Busquets, Jaume (2004). El Paisaje como Oportunidad. En Portada. CEIDA. Centros de Educación e Investigación Didáctico Ambiental. En: http://www.euskadi.net/vima educacion/indice c.htm

- Dirección de Planeamiento Urbano (1995). Plan hídrico ambiental. Primera Fase. Etapa de Consulta. Diagnostico Físico. Subsecretaría de Planeamiento y Desarrollo Urbano Ambiental. Resistencia. Chaco. En:http://hum.unne.edu.ar/academica/institutos/geografia/default.htm

- Escudero Gallegos, Carlota y Guevara Senciales, José (1991). Los problemas ambientales en las concentraciones urbanas. Segundo Congreso Internacional de Ordenación del Territorio. Valencia (España). pp. $223-239$.

- Fernández García, Felipe y Vidal Domínguez, María Jesús (1991). Los impactos ambientales en la ciudad. El caso de Madrid. Segundo Congreso Internacional de Ordenación del Territorio. Valencia (España). pp. $241-254$.

- Ferrer Regales, Manuel (1992). Los sistemas urbanos Espacios y Sociedades, Serie General №14, Madrid, Síntesis.

- Folch, Ramón (2007). El espacio territorial metropolitano, un bien escaso. En: $\quad$ http://www.erf.cat/php/cas/print.php?id text=142

- Foschiatti, Ana Maria. (2004). Vulnerabilidad Global y Pobreza. Consideraciones conceptuales. En: Revista Geográfica Digital. IGUNNE. Año 1. № 2. Julio - Diciembre.

- Foschiatti de Dell'Orto, Ana M. (1992). El desarrollo urbano y las particularidades demográficas del Chaco y su capital entre 1960 y 1990. Geográfica № 115, IPGH, México.

- Gatica, Fernando (1980). La urbanización en América Latina: 1950-1970; patrones y áreas críticas. En: Joop, Albert y Villa, Miguel. Redistribución espacial de la población en América Latina, CELADE, Santiago de Chile. 
- George, Perre (1972). El Medio Ambiente. Traducción de A. Giralt Pont. Ed. Península. Historia, Ciencia y Sociedad No 61. Barcelona (España).

- Gottmann, J. (1961), Megalopolis; the urbanizet northeastern seaboard of US, Cambridge (MA): MIT Press.

- Hardoy, Jorge E. y Morse, Richard M. (1988). (comp.) Repensando la ciudad de América Latina. Buenos Aires, Grupo Editor Latinoamericano S.R.L.

- Hardoy, Jorge E. y Schaedel, Richard P. (comp.) (1975). Las ciudades de América Latina y sus áreas de influencia a través de la historia. Buenos Aires, Ediciones SIAP.

- Herrera, Ligia (1976). La concentración urbana y la dispersión de la población rural en América Latina. Su incidencia en el deterioro del medio humano. Serie A, NN136, CELADE, Santiago de Chile. En: http://habitat.aq.upm.es/boletin/n15/ajfar.html

http://www.saniplan.org/startspa.htm.

- Huaman, L. (1947-50). La Vegetación de la Argentina. En Geografía de la República Argentina. Ed. Sociedad Argentina de Estudios Geográficos, Bs. As. Tomo VIII.

- Manoiloff, Raúl O. A. (2000) Caracteres del Movimiento Natural de la Población del Gran Resistencia en 1970, 1980 y 1990. En Nordeste. Segunda época. Serie Investigación y Ensayo. 13. Geografía. Fac. Humanidades. UNNE

- Matteucci, S.D.; J. Morello; A. Rodríguez; G.D. Buzai y C. Baxendale. (1999). El crecimiento de la metrópoli y los cambios de biodiversidad: el caso de Buenos Aires. En: S.D.Matteucci; O.T.Solbrig; J.Morello y G.Halffter. 1999. Biodiversidad y uso de la tierra. Conceptos y ejemplos de latinoamérica. EUDEBA-UNESCO, Buenos Aires. Pp. 549-580.

- Melnechuk, Paula V. (2001). La Defensa del Ambiente Frente a las Amenazas de Impacto. El caso de la laguna Argüello de Resistencia. Monografía. Maestría en Gestión Ambiental. F.A.U. UNNE. Resistencia. Chaco. Pag. 10

- Miranda, Guido (1955). Tres Ciclos Chaqueños. Crónica Histórica Regional. Ed. Norte Argentino. Resistencia,

- Montenegro, Raúl (1998). Ecología de sistemas urbano. Documento del curso: "La Gestión Ambiental en el Desarrollo Urbano". Maestría de Gestión Ambiental del Desarrollo Urbano GADU. Facultad de Ingeniería. Universidad Nacional del Comahue. Neuquén.

- Morello, J.; S. Matteucci; G. Buzai; A. Rodríguez y C. Baxendale. (2000). Cambios Ecológicos en las fronteras urbano rurales: el caso de la región metropolitana de Buenos Aires. Segundas Jornadas Platenses de Geografía: Resignificando una Geografía para Todos. Universidad Nacional de la Plata, La Plata.

- Morello, Jorge y Adámoli, Jorge. (1967). Vegetación y Ambiente del Nordeste Argentino del Chaco Argentino. Boletín № 3 de Estación Experimental Agropecuaria de Colonia Benítez. INTA.

- Morello, Jorge y Otros (Febrero / Marzo 2000). Urbanización y consumo de tierra fértil. En Ciencia Hoy. Volumen 10, № 55, pp. 50-61.

- Muscar Benasayag, Eduardo F. y Franchini; Teresa. (1992). Emplazamiento Urbanos en Zonas de Riesgos naturales: El Caso del Gran Resistencia en la Planicie Chaqueña. En Estudios Geográficos. C.S.I.C. Centro de Investigaciones Sobre la Economía, la Sociedad y el Medio (C.I.ES.M.) Madrid. LIII, 208. Julio-Septiembre, pp. 481 a 501.

- Nuestra Arquitectura (1980). "Plan para el Ordenamiento Ambiental de Resistencia y sus áreas de influencia", № 508.

- Popolizio, E; Serra, P. y Hort, G. (1978). Fotointerpretación Aplicada al Estudio de la Cuenca del Río Negro - Provincia del Chaco. Tomo 14. Centro Geociencias Aplicadas. Serie C Investigación. Facultad de Ingeniería y de Humanidades. U.N.N.E. Resistencia - Chaco, Argentina.

- Reboratti, Carlos (1999). Estructura y dinámica del territorio. Documento del modulo 4. Maestría de Gestión Ambiental. Facultad de Arquitectura y Urbanismo. UNNE. Resistencia. Chaco.

- Rechini de Lattes, Zulma (1973). Aspectos geográficos de la urbanización en la Argentina. 1869-1960. Buenos Aires, Centro de Investigaciones Sociales - Instituto Torcuato Di Tella, CELADE. 
- Rey, Walter (1997). La Organización del Espacio y Algunas Consideraciones Ambientales de Resistencia y Presidencia Roque Sáenz Peña. En Revista Nordeste. № 5. Segunda Época, Geografía. Serie Investigación y ensayo. Fac. Humanidades, UNNE, Resistencia, pp. 167 a 185.

- Riba, Jorge Ricardo (2007) Hay que Diseñar con un Estilo Propio. Globalización, arquitectura, urbanismo y pobreza. Prensa.com. Panamá, viernes 27 de julio de 2007. En http://mensual.prensa.com/mensual/contenido/2007/07/27/hoy/opinion.shtml

- Roccatagliata, Juan (1999). Ordenamiento Territorial y Sistemas de Ciudades. Documento del curso: "La Gestión Ambiental en el Desarrollo Urbano". Maestría de Gestión Ambiental del Desarrollo Urbano GADU. Facultad de Ingeniería. Universidad Nacional del Comahue. Neuquén.

- Rodríguez de Sancho, María Jesús (1991). Los problemas ambientales en las concentraciones urbanas. Segundo Congreso Internacional de Ordenación del Territorio. Valencia (España). $1763-1776$.

- Rufí, Joan Vicente (2000) ¿Nuevas palabras, nuevas ciudades? En: Revista de Geografía. (ISSN 0048-7708) Universidad de Barcelona. № 2. 2003 / 79-103

- Sáez, María B. (2003) Resistencia. Algunas cuestiones puntuales. FAU. UNNE. Resistencia. Chaco

- Sassone, Susana M. (2000). Reestructuración Territorial y Ciudades Intermedias en la Argentina. En Ciudad y Territorio. Estudios Territoriales, XXXII (123). Ministerio de Fomento. Madrid (España). pp. 57-92.

- Scornik, Carlos (1998.). Diagnóstico Expeditivo AMGR.- Ministerio del Interior- Programa de Protección Contra Inundaciones (P.P.I).-SUPCE- Convenio BIRF- Provincia del Chaco 364p.

- Serra, Pilar Yolanda (Agosto 1995). El Sitio Urbano de Resistencia, Barranquera y Vilelas. En Resúmenes II Encuentro de Profesores de Geografía de Formosa,, p.p. 63 a 69.

- Smith, Neil. (1996). The new urban frontier. Gentrification and the revanchist city, Londres: Routledge

- Subsecretaría de Ordenamiento Ambiental. Gobierno de la Provincia del Chaco. (1980). Plan de ordenamiento ambiental de Resistencia y su área de influencia. Separata de Nuestra Arquitectura, № 508.

- Tella, Guillermo (2007). Soluciones para metrópolis en Problemas. En: Noticias. Universia. Argentina. Red de Universidades, red de oportunidades. (Publicado el 06/06/2007. En: $\quad$ http://www.universia.com.ar/landings/noticias.html

- Tello, Enric (1999). La formación histórica de los paisajes agrarios mediterráneos: una aproximación coevolutiva. En Historia Agraria. Ed Seha. № 19, pp. 195-212. Granada.

- Tojo, José Fariña. (2001) Naturaleza urbana. En Boletín CF+S. Número 15. Marzo. Ed. Instituto Juan de Herrera. Madrid.

- Valenzuela de Mari, Cristina (1991). Los problemas de la expansión urbana y demográfica de la ciudad de Resistencia, desde la perspectiva geográfica, a partir de 1960. Cuadernos de Geohistoria Regional No 23. IIGHI. Resistencia.

- Vinuesa Angulo, Julio y Vidal Domínguez, M.J. (1991). Los procesos de urbanización. Espacio y Sociedades № 3, Síntesis, Madrid.

- Zárate, Antonio (1992). El mosaico urbano. Organización interna y la vida en las ciudades. Colombia, Cincel - Kapeluz. 
